\title{
Die Nervenendigungen in der Haut der äusseren Genitalorgane des Menschen.
}

Von

\author{
A. S. Dogiel, \\ Professor der Histologic an der Universität Tomsk (Sibirien).
}

Hierma Tafel XXXII und XXXIII und ein Holzschnitt.

W. Krause und scine Schüler Polle, W. Finger und Bense haben bekanntlich zuerst darauf aufmerksam gemacht, dass in der Haut des Penis und der Clitoris beim Menschen und bei den Siiugethieren Nerrenendapparate sich vorfinden, und zwar kugelige Endkolben und besondere Körperchen, welche man Genitalnervenkörperchen (Wollustkörperchen) nannte. Seit dieser Zeit sind viele Arbeiten über Nervenendigungen in den Genitalnervenkörperchen, wie von A. Key und G. Retzius, Izquierdo, Merkel und zuletzt Aronson, Schwalbe und G. Retzius erschienen, von welchen besonders die vou Aronson und G. Retzius beachtenswerth sind, zumal von diesen Forschern zuerst das neue Mittel zur Nerventinction, das Methylenblau, angewendet wurde.

Als Object seiner Untersuchungen nahm H. Aronson die Haut der änsseren Genitalien (Penis, Clitoris und Vagina) des Kaninchens, in welcher man nach seinen Beobaehtungen zweierlei Typen von Genitalnervenkörperchen unterscheiden kann - grosse und kleine; die letateren trifft man hauptsächlich in der Schleimhaut der Clitoris und der Vagina an. In die grossen Körperchen treten gewöhnlich mehrere Nervenfasern ein, welche sich in jedem Körperchen auf verschiedene Weise krümmen und verwickeln, unterwegs viele, mit einander sich verbindende Seitenästchen aussenden und damn in kleinen knopfförmigen Anschwellungen endigen. Diese Endanschwellangen sind nach Angabe H. Aronson's oft mit einander durch Anastomosen verbunden. Was die kleinen Körperchen anbetrifft, so empfangen sie stets nur eine Nervenfaser, welche nach dem Eintritt in das Körperchen in eine gewisse Anzahl feiner Aestchen zerfällt, die sich wiederum mehrfach theilen und in Endknöpfehen endigen.

Archiv f. mikrosk. Anat. Bd. 41 
G. Retzius untersuchte die Nervenendigung ähnlich wic Aronson in den Genitalnervenkürperchen der Hant des Penis und der Clitoris beim Kaninchen, indem er intravitale Injection mit Methylenblan machte (1 gr auf $400 \mathrm{ccm}$ einer $0,75 \%$ Kochsalylïsung). Nach den Beohachtungen von G. Retzius treten die markhaltigen Nervenfasern an die Körperchen heran, indeu sie sich oft dichotomiseh in einige (2-3) Aestehen theilen und verlieren grösstentheils in der Nähe dieser Körperehen ihre Markscheide. In einigen Fällen verliert die Nervenfiser ihre Marksubstanz noch vor dem Eintritt in das Körperchen und theilt sich daranf in zwei Aestehen, von denen jedes in zwei neben einander liegende Körperchen cintritt.

Nachdem die Nervenfaser in das Körperehen eingetreten, zerfällt sie nach Retzins's Ingaben in zwei ziemlich dicke Aestehen oder gieht nach verschiedenen Richtungen feine Zweigchen ab und verläult damn weiter, sich windend, in dem Axentheil des Körperchens, wobei sie während dieses Verlanfs fortfaihrt beständig ant's nene Aestehen ahzugeben. Dasselbe findet anch in dem Falle statt, wenn sich die Nervenfaser dichotomisch getheilt hat: nach dem Eintritt in das Inncre des Kirperehens zerfällt jeder der Aeste, je mach der Grösse des Könnerehens, in cine mehr oder weniger grosse Anzall feincr Aestchen, welche, sich verschiedentlich schlängelnd, nach dè obertliehlichen Particen des Körperchens hinziehen. Nach vieltacher Theilung endigen alle Nervenzweige in der Nähe der Oberfliche des Körperchens in kleinen knoptähhnlichen, rundlichen, birnförmigen oder ovalen Anschwellungen (Endknöpfehen), oder zureilen olme jegliche Anschwellung. Anastomosen zwischen den knopfförmigen Anscbwellnngen, wie sie Arouson beschrieben, so wie auch feine Verzweigungen, welche sich mit andercn Aestchen in Verbindung setzen, hat Retzius nicht beobachtet. Die Form und Grösse der Genitalnervenkörperchen ist nach Retzius eine' sehr verschiedene und neben kleinen Körperchen ron ovaler, bisquitahnlicher oder unregelmässig gestalteten Form trifft man bestïndig auch grössere von derselben Form, hälffig eingeschnürte and in mehrere Abtheilungen zerfallende Körperehen an; die Nervenfaser theilt sich gewöhnlich beim Ferantreten zu den letzteren in eine entsprechende Anzahl Aestchen, die sich nach jeder Abtheilung linbegeben. 
Die Nervenendigungen in der Iilut der itusseren Genitalorgane ete. 587

Auf solche Weise stimmen also sowohl die Beobachtungen von H. Arouson wie die von G. Retzius darin überein, dass die Nervenfaser im Imern des Genitalnervenkörperchens in eine gewisse Anzahl feiner Zweige zerfällt, welche in knopfförmigen Anschwellumgen frei enligen, ausserdem aber nimmt Arouson noch an, dass zwischen den einzelnen Nervenästchen und sogar zwischen den Endknöptehen Anastomosen vorhanden sind, deren Existenz Retzius verneint.

In letzter Zeit habe ich selbst die Möglichkeit gefunden, dias Verhalten der Nerven zu den Genitahervenkörperchen beim Mcnschen zu erforschen, woriiber ich die Resultate meiner Bcobachtungen in vorliegender Mittheilung darzulegen beabsichtige.

Die Färbung der Nerven in der Haut der inneren Lamelle des Praeputium, Frenulum pracpntii, Glans penis, Fossa navicnlaris und Clitoris des Menschen geschah mit $1 / 1 \mathrm{ti} \%$ Lösung von Methylenblau mach der von mir bereits beschriebenen Methode auf dem Objecttrïger; die der Färbung unterworfenen Theile der Schleimhaut hatten eine Länge von circa 2-3 $\mathrm{cm}$ und eine Breite von $1-2 \mathrm{~cm}$.

Zur Fixirung der Färbung der Nervenelemente wurde die Haut auf 24 Stunden in eine gesättigte wässerige Lösnng von Anmoniumpikrat oder in eine Ammonium-Pikrat-OsmiumsäirreMischung gelegt; darauf (in der Mehrzahl der Fälle) wurde das Epithel von der Oberfläche vorsichtig entfernt, wobei die letztere auf dem Objectträger sorgfältig ansgebreitet und in Glycerin eingebettet wurde. Nach einigen Tagen war das Präparat vollständig durchsichtig und zur Untersuchung geeignet geworden. Die Schnitte wurden ausschliesslich von der Haut der Glans penis entnommen, deren Nerven zuvor mit Methylenblau gefürbt und mittelst oben angeführter Mischung fixirt waren, wonach kleine Stückchen der Schleimhaut an Hollundermark anfrieren gelassen und darauf mit dem Rasimesser ouler mit dem Mikrotom geschnitten wurden.

An mit schwachen Objectiven zu untersuchenden Schmitten ist ersichtlich, diss in der tiefsten, mehr Inckeren Schicht der Haut cine Menge Nervenstïmmehen von rerschiectener Dicke sich 
vorfinden, welche, unter einander anastomosirend, einen breitmaschigen Nervenplexus bilden (Fig. 1c). Von dem letzteren theilt sich eine beträchtliche Anzahl feiner Nervenstämmchen ab, welche in schrüger orler senkrechter Richtıng in die melır oberflächliche und dichtere der Pars reticularis corii entsprechende Hautschicht eintreten, wobei sie nnterwegs kleine Zweige an die benachbarten Stämmchen abgeben und schliesslich selbst, sich allmählich der Wärzchenschicht nähernd, in einzelne mehr oder weniger feine Aestchen zerfallen. Einige der eben bezeichneten feinen Aestchen, wie unten geschildert sein wird, betheiligen sich vollständig an der Bildung der Nervenendapparate, wälsrend audere zuvor in einzelne Fasern zerfallen, welche daranf zum Theil in den Endkörperchen, zum Theil im Epithel endigen.

An der Bildung sowohl dicker wie dïnner Nervenstämmchen und Aestchen betheiligen sich vorzugsweise markbaltige und nur eine geringe Anzall markloser Nervenfasern. Markhaltige Fasern, welche sich in den Nervenstämmchen einlagern, theilen sich, insbesondere an Verzweigungsstellen der letzteren, viclfältig in 2-3-4 Fasern, die eine gewisse Strecke im Stämmchen selbst durehmachen und sodam aus demselben austreten, un schliesslich in Krumunngen in die Haut überzugehen. Von den ebenbezeichneten Aestchen, die durch Theilung der Nervenfascrn entstanden sind, werden häufig marklose Aestchen abgesondert, welche ihrerseits, ehe sie die Bildung dler Nervenendapparate bewirkt haben, sich vielfach wiederum theilen können. In vielen Fällen gehen von irgend einer markhaltigen Faser, die in dem Stämmchen sich einlagert, an der Stelle des Ranvier'schen Schnurringes ein, ja zuweilen sogar zwei feine marklose Aestchen aus, welche häufig eine beträchtliche Strecke weit in dem Nervenstämnichen selbst. verlatien und sehr leicht mit den marklosen - Remak'schen Nervenfasern verwechselt werden können.

Die Mehrzahl der Nervenstämmehen und einzelner markhaltiger Fasern, welche aus dem oben beschriebenen Nervenplexus entstanden sind, endigt in der Hant der inneren Lamelle des Praeputium, Frenulum praeputii, der Glans penis, Fossa navietlaris und Clitoris in Nervenendapparaten versehiedener Art und "war: a) in Genitalnervenkörperchen, b) in Nervenendkörperchen - Endkolben, W. Krause - und c) in Meissner'schen 'Tastkïrperehen. 
Die Nervenendigungen in der Haut der äusseren Genitalorgane etc. 589

a) Die Genitalnervenkörperchen (Fig. 1-11) beim Mensehen liegen in einiger, bald mehr, bald weniger betrïchtlichen Entfernung von der papillentragenden Schicht der Haut und werden niemals im Gewebe der Wärzchen selbst angetroffen (Fig. 1d). Einzelne Körperchen findet man zuweilen in jener lockeren und tiefen Schicht, in welcher, wie oben erwïhnt, auch die dicken Nervenstämmehen Platz gegriffen haben. Die Form der Genitalnervenkorperchen ist, wie tronson, Schwalbe, Retzius und Andere ganz richtig bemerken, eine sehr mannigfałtige: rund, oval, ei- nnd birnförmig; einige dieser Körperehen crscheinen als stark in die Länge ausgezogene oder cylinderförmige Bildungen. Oft werden von irgend einem der Körperchen Anhängsel verschiedener Grösse und Form abgesandt, wodurch das Körperchen eine unregelmässige, sprossenartige Gestalt annimmt. Zuweilen erscheint das Körperchen gekrummit oder an irgend einer Stelle mehr oder weniger stark eingeschnurt. So viel ich beobachten konnte, trifft man am häufigsten runde, ovale und eiförmige Kürperchen an. Gewöhnlich sind die Körperchen ziemlich nahe bei einander gruppirt und dabei in der Art, dass ihr Längendurchmesser mehr oder weniger parallel der Oberfläche der Haut belegen ist (Kig. 1d). Die Anzahl der zu beschreibenden Körperchen ist offenbar eine sehr betrïchtliche, da man in einem Theile der Ilaut der Glans penis von dem Umfang eines Quadrat-Willimeters annähernd 1 bis 4 Körperchen antriftt und zuweilen auch mehr; in der Haut des Praeputiun und Frenulum praepntii findet man dergleichen Körperchen dem Anscheine nach weniger als in der der Glans penis und Clitoris.

Was die Grösse der Genitalnervenkörperchen beim Henschen aubetrifft, so kann sie ebenso wie ihre Forn eine verschiedene sein: neben kleinen Körperchen von 0,04-0,09 mm Breite und 0,06--0,12 mm Länge, begegnet man Kürperchen von beträchtlicher Grösse, deren Länge zwischen 0,13-0,20 mm schwankt, während die Breite 0,07-0,20 mm betrügt; manche Körperchen erreichen eine Länge ron $0,40 \mathrm{~mm}$, bei einer Breite von $0,10 \mathrm{~mm}$.

Ein jedes Körperchen, wie bereits ron W. Krause, W. Finger, Merkel, Izquierdo, Schwalbe und G. Retzius angegeben wurde, ist von einer Bindegewebshïlle umgeben, welche eine beträchtliche Dicke und Consistenz besitzt und eine grosse Anzahl Kerne enthïlt. Niach der Besehreibung ron 
Schwalbe bestelit diese Iülle ans Endothelhäntchen, welche den Innenkolben concentrisch umgeben, wobei zwischen den äusseren Kapselsystem der Hiille und dem Imnenkolben eine Schicht grosser flacher Kerne sich vorfindet. An den Schnitten der durch Flüssigkeiten verschiedener Art crhärteten Haut und an mit Essigsämre behandelten Prïparaten wurle von G. Retzius beobachtet, dass zwischen der innersten Kapsellamelle des Kïrperchens und dem Innenkolben Gebilde von rundlicher, ovaler odcr eckiger Form wahruchmbar sind, welche in einer schwach kürnigen Masse ihren Sitz gefunden haben. Die bezeichneten Gebilde hält Retrins für diejenigen Kerne, welche Schwalbe bei seincr Beschreibung der Hülle der Genitalnervenkörperehen erw:ihnt.

Ich habe die Kapsel der Körperchen an Schnitten der II int (der Glans penis) untersucht, die zuvor mit Methylenblau sefüirbt und durch Ammoniumpikratlösung ofler durch Aumonium-PikratOsminmsäture-Hisehmg fixirt wurle, wobei zur Färbung der Schnitte das Hoyer sche Pikrokarmin Anwendung fand. In einigen Fiallen konnte man rermittelst selwwacher Objective in den durch Methylenblau gefärbten, fixirten und schliesslich durch Glycerin geniigend aufgrehellten Antheilen der Haut grosse Genitalnervenkörperchen wahmehmen und sie dam mit cinem geringen Theil des sie umgebenden Bindegewebes vorsichtig mittelst einer kleinen Scheere ausschneiden; die auf solche Weise herausgeschnittenen Theilchen wurden auf dem Objektträger mit dem Hoyer'schen Pikrokarmin tingirt und mit Glycerin behandelt.

Durch Anwendung des eben beschriebenen Verfihrens gelang es mir oft, die Genitalnervenkörperchen fast vollständig zu isoliren und Dank diesem Umstande den Ban der sie umgebenden Hülle genau zu erforschen.

Um die Hülle der zu beschreibenden Körperchen zu untersuchen, wurde ausserdem die Hant der Glans penis durch Fliissigkeiten verschiedener Art (M ïller'sche Flïssigkeit, SublimatLösung, Flemming'sche Mischung u. s. w.) gehärtet, worauf aus derselben Schnitte gefertigt und diese mit irgend einem Färbmittel tingirt wurden.

Auf den nach einer der chen angefïhrten Methoden behandelten Prïparaten tritt einerseits die Hülle der Körperchen sehr deutlich hervor, andererseits ist der Innenkolben mit dem 
Die Nervenendigungen in der Haut der iunsseren Genitalorgane etc. 591

in demselben cingeschlosscuen Nervenendapparat vorzïglich deutlieh $x a$ erkennen.

Gewöhnlich unıgibt die Hülle jedes einzelne Körperchen und hat je nach rer Grösse resselben eine mehr oder weniger betrïchtliche Dicke: an grossen Körperchen erreicht diese Dicke zuweilen von $0,02-0,03 \mathrm{~mm}$, an kleinen Körperehen pHegt sie viel geringer zu sein. Die Hülle besteht ats einer ganzen Reihe feiner bindegewebsartiger Häntehen, welche Längsstreifungen darbieten und, sich concentrisch über einander schichtend, den rund, oval odler urregelmässig geformten Innenkolben des Kürperchen einsehliessen.

Die Anzihh der IÏ̈ntchen, welehe die Itülle bilden, ist direct von der Dicke der letzteren abhänsig und beläuft sich von 3- $t$ auf $6-8$ und mehr. In der Mehrzabl der Fäle kamn nuan an den sohnitten und an den isolirten Körperchen wahrnehmen, diss ein Häntchen sich eng an das andere benachbarte anschliesst, indem es von dem letzteren durch eine Schicht unregehmïssig. gestalteter flacher Zellen mit grossen runden odler ovalen Kemen getrennt ist, wodurch die Hülle der Cenitahervenkürperehen einige Achnlichkeit mit đer Hülle der Pacini sehen Kür'perehen erlaugt (Fig. 2). Die Oberflïehe des innersten Häutchens, welehes nach der Iühlungsseite des Körperchens gerichtet ist und dieselbe begrenzt, ist mit einer Schicht (ler eben beschriebenen flachen Zellen belegt (Fig. 2), deren kernhaltiger Theil mehr oder weniger tief in den Innenkolben hineinriuckt, wie es bereits zum Theil schwalbe und darauf in letzter Zeit G. Retzius beschrieben haben.

Wie die Nerven der Genitalnervenkörperchen der Haut der Glans penis und der Clitoris beim Menschen endigen und wie der Bau des Innenkolbens beschaffen ist - in dieser Hinsicht sind unsere Kenntnisse noch sehr beschränkt. Nach der Schilderung von W. Krause und W. Finger treten in den Innenkolben jedes Körperehens eine, zwei oder mehrere Nervenfasern ein, welche darauf sich mehrfach in feine Terminalfasern theilen, wovon auch das feinkörnige Aussehen des Innenkolben-Inhaltes abhängig ist. Es ist G. Retzius nicht gelungen, die Endigungen der Nervenfasern in den Genitalnervenkörperchen beim Mensehen zu erforschen. Schwalbe stimmt mit W. Krause darin überein, dass die Nerventasern, nachdem sie in den Innenkolben einge- 
treten, in Terminalästchen zerfallen, welche aller Walurscheinlichkeit nach als knopfförmige Ansehwellungen endigen, wobei zutgleich auch eine Theilung des Innenkolbens selbst stattfindet, so dass jedes Tervenendfüserchen sich in einen besonderen Imnenkolben einlagert. Anf solche Weise muss ter Bau des Innenkolbens der bezeichneten Körperchen des Menschen nach der Yeinung von Schwalbe ganz derselbe sein, wie der Bau der runden Endkolben.

Veine eigenen, diesen Gegenstand betreffenden Beobachtungen, welehe ich an den Genitalnervenkörperchen des Menschen anstellte, erweisen, dass die Imzahl der Nervenfaserm, welche in ein Körperchen eindringen, in ciner gewissen Bezichung nur Grösse des letzteren steht: ein kleines Körperehen erhält meistentheils eine, zwei, selten drei markhaltige Nervenfasern (Fig. 1,, , \pm und 11), während in die grossen Kürperehen 3 his $8-10$ Nervenfasern eintreten (Fig. 1, 3, 7 und 10). Dic Eintrittsstelle der letzteren in die kleinen Körperchen ptlegt eins der Polenden derselben oder irgend eine stelle an der Obertliche des Kïrperehens zu sein. In einigen Fällen treten die Nerrentasern in irgend ein kleines Körperchen an beiden Polenden desselben cin. Ausserdem kann man oft heobachten, dass der Axencylinder der markhaltigen Faser, nachdem dicse ihre Varkscheile noch vor dem Eintritt in rieses odler jenes Körperchen verloren hat, sich in einige (2-3) destchen theilt, welche sich nach einem der Körperchen hinbegeben (Fig. 4 md 6), oder ein oder zwei lestchen treten in ein Körperchen ein, während die übrigen uach einem anderen, benachbarten Körperchen sich begreben, oder schliesslich, jedes Aestchen nimut seine Richtung nach einem abgesonderten Körperchen. Oft macht eine marklialtige Faser ein oder zwei Windungen rings um das kleine Köruerchen und tritt erst dam in den Innenkolben ein, wobei sich zuweilen von dem Axencylinder einer solchen Faser an der Stelle der Ranvier'schen Schnürringe ein oder zwei marklose Aestchen abtheilen. Nachdem die letzteren eine kurze Strecke weit an der Oberfläche des Körperchens sich hingezogen, durchdringen sie die Hiulle desselben und werden num ebenfalls zur Bildung des Nervenendapparats rerwanclt.

Was die grossen Genitalnervenkörperchen anlelingt, so treten gewöhlich alle Nervenfisern, mit welehen ein solches 
Die Nervenendigungen in der Haut der äusseren Genitalorgane ete. 593

Körperchen versorgt wird, an dasselbe von verschiedenen Seiten heran, und zwar in der Form von abgesonderten Fasern (Fig. 1, 4, 6, 7 und 10) oder zu 2-3 liasern zusammen, oder endlich zuweilen als ein oder awei Stämmchen, von denen jedes aus $\tilde{0}-6$ Fasern besteht (Fig. 3). In dem Falle, wemn das Körperchen stark in die Länge gestreckt erscheint, treten die Nervenfasem von beiden seiner Polenden in dasselbe ein (Fig. 7). Oft theilt sich eine oder die andere der Fasern, welche die Richtung nach einem der Körperchen genommen, in der Nähe von dessen Hülle in einige testchen, von denen manche markhaltig, andere aber marklos sind.

Wem die Nervenfasern die Hülle erreicht haben, winden sie sich an deren Oberflizehe, viele von ihnen theilen sich an der Stelle der Ranvier'sehen Einschniirungen dichotomisch, durchkreuzen sich gegenseitig in verschiedenen Richtmngen und uiberspimnen schliesslich das Körperchen von allen Seiten, wie dieses aus Fig. $t$ ersichtlich ist. Nacholem die Nervenfasem un das betreffende Körperehen eine gewisse Anzahl Windungen gemacht haben, verlieren sie ihre Marksubstanz, durchdringen in verschicdenen Punkten als nackte Axencylinder die Hülle des Endapparats und treten in den Innenkolben des Kürperchens ein (Fig. 4).

Wenn die Nervenfasem, welche die Richtung nach irgend einem Körperchen genommen haben, in Nervenstämmchen eingeschlossen sind, verlieren gewöhnlich einige derselben ihre Markscheide in der Nähe des Körperchens oder anf dessen Oberfläche (Fig. 3) und nachdem die Fasern sich in einige Aestchen getheilt haben, machen sie um das Körperchen ein, zwei Windungen, wïlurend andere Nervenfasern sogleich nach dem Verlust ihrer Marksubstanz in den Innenkolben eintreten. Man trifft zuweilen soleher Art grosse Körperchen, welche 2-3 dicke Nervenfasem empfangen, wie dieses aus Fig. 8 ersichtlich ist; nachdem die letzteren an die Hülle des Körperchens herangetreten sind, verlieren sie ihre Marksubstanz und dringen in Form sehr dicker Axencylinder in den Innenkolben ein. Ausserdem kommt es nicht selten vor, dass ron irgend ciner Faser, welche das eine oder das andere der Körperchen umwindet, an der Stelle der Ranvier'schen Einschnürung zwei kurze marklose Aestehen sich abtheilen, wïlurend von einer anderen Faser ein mehr oder weniger langes Aestehen ausgesentet wird, das mit Markscheide rersehen 
ist, wobei die ersteren wie anch die letzteren ahgesonderte Endapparate bilden (Fig. 4A), welche neben dem betreffenden grossen Kürperchen gelegen sind.

Unı das eben geschilderte Verhalten der Nervenfasem zn den Genitalnervenkïrperchen beobachten zu kïnnen, ist es am geeignetsten, in dem Präparat solehe Körperehen auszusuchen, deren Endapparate entweder gar nicht oder nur zum Theil mit Methylenblau tingirt worden sind, weil bei vollständiger Färbnng aller in Iunenkolben befindlichen Nervenistchen und Fäden es viel sehwerer hält, den Verlauf' der an der Obertläche des Körperchens sich windenden Nervenfasem zu vertolgen.

Was dic'Sehwann'sche Scheide so wie die bindegewebige Hülle, welehe die an die Körperehen herantretenden Nervenstämmehen umgeben, ambelangt, so gelit gewöhulieh die crstere wie anch die letztere noch ror dem Eintritt der Axencylinder der Nervenfasern in den Innenkolben, in dic Hülle des Körperchens iiber (Fig. 2 und 3).

Sobald ein oder mehrere Axencylinder der Nerventisern in den Innenkolben des Körperehens eingetreten sind, bilken sie. heim Einrücken in den peripheren oder meh" centralen Theil des Kolbens in demselben eine grössere oder kleinere Inzahl bogenförmiger schlingen, und senden unterwegrs mach verschiedenen Richtungen allmählich zablreiche Aestchen von versehiedener Länge und Dicke ans (Eig. 2, 3, 5, 6, 7, 8, 10 und 11). Nachlem die Axencylinder schliesslich oft eine beträchtliche Strecke weit im Innenkolben verlanfen sind und in demselben eine oder zwei Umwindungen gemacht haben, zerfallen sie endlich in einige mehr oder weniger feine destchen. Zuweilen findet die Theilung des Axenicylinders sogleich nach dem Eintritt desselben in den Innenkolben statt.

Alle zahlreichen Nervenästchen, welche sich durch allmähliche Theilung der in das Innere der Körperchen eindringenden Axencylinder der Nervenfasern gebildet haben, machen Windungen und verflechten sich untereinander nach verschiedenen Richtungen hin; während des Verlaufs zerfallen sie je nach der Grösse des Körperchens in verschiedene mehr oder weniger zahlreiche feine Alestchen und Fäden, die sich in zickzackförmigen Windungen schlängeln, durchkreuzen nnd mit einander in der allermannigfaltigsten Wcise verflechten, so dass eine genalue Beschreibung. 
Die Nervenendigungen in der Haut der äusseren Genitalorgane etc. 595

fast unmöglich ist; dabei sind sie wiclerum der Theilıng in zahlreiche teine und sich mit einander verflechtende Fäden unterworfen (Fig. 1-11). Ein grosser Theil der soeben beschriebenen Aestchen und Faiden vereinigt sich mit anderen benachbarten Fäden und bildet ein dichtes Netz ${ }^{1}$ ), während ein anderer geringerer Theil allem Anscheine nach mit Anschwellungen von versehiedener Grösse und Form endight, die sich entweder an der Peripherie des Innenkolbens befinden oder in Imnern des letzteren zwisehen den dureh die Nervenästehen gebildeten Schlingen ilren Sitz haben, wobei einige dieser Anschwellungen sich unnittelbar an die erwähnten Aestchen anlegen (Fig. 2-8). Man kann ausserdem sehr oft wahrnehmen, dass aus dem einen oder dem auderen Zweigchen oder Farlen kurze Seitenfäden ausgehen, von denen die einen mit benachbarten Fäden verbunden sind, andere wicdernm mit Anschwellungen endigen (Fig. 7 and 8).

Die Endanschwollungen haben bald eine runde, ovale, birnförnige oder spindelartige Form, bald erseheinen sie im Gegentheil in der Form dünner Plättchen mit zackigen Ründern oder als unregelnüissig geformte polygonale, abgeplattete Gebilde, wobei ihre Grüsse gleich ihrer Form variirt: neben selur kleinen finden wir stets auch ziemlich grosse Anschwellungen (Fjg. 7 u. 8). Wenn man die eben beschriebenen Endanschwellungen mit einem starken Objective (1/12 Homog. Imm. Syst. Zeiss) untersucht, so kann man sich davon iuberzengen, dass von den Rändern und Winkeln einiger derselben sehr feine Fäden ausgehen, welche sich mit benachbarten Aestchen und Fäden vereinigen. Folglich haben die oben bezeichneten Anschwellungen in der That uicht den Charakter von Endanschwellungen.

Bei mögliehst sorgfültiger Untersuchung einer Henge von Präparaten beobachtete ich eine sich stets wiederholende Erscheinung, nämlich: je vollkommener und vollständiger die Tingirung der Nervenästchen und Fäden war, eine desto geringere Anzahl jener oben beschriebenen freien Endignngen war in den letzteren anzutreffen, und im Gegentheil, bei nicht vollkommen ge-

1) In Anbetracht dessen, diss alle Nervenistchen und Fiden aus Nervenfibrillen bestehen, halte ich das Nervennetz nicht für den Zusammenflusspunkt der genannten Aestchen und Fäden, sondern ich verstehe unter der Bezeichnung Netz nur den Ort, wo eine Verflechtung der Nervenfibrillen stattindet. 
lungener Tingirung erschien ihre Anzahl weit beträchtlicher, wobei man in solchen Fällen ein Bild erhielt, das den Zeichnungen entsprach, die der trefflichen Arbeit von G. Retzius beigegeben sind. In einzelnen Fällen konnte man bei sehr vollständiger Färbung des Nervenapparats in keinem der Körperchen unieachtet der allersorgfültigsten Untersuchung anch nur cine cinzige Endanschwellung finden; dabei ist die Anzahl der Nervenfäden und der von ihnen im Innenkolben gebildeten Schlingen und Windungen eine so grosse und sic legen sich so eng aneinander an, dass in der Höhlung des Körperchens in der 'That keine Stelle übrigg erscheint, welche nicht von ihnen eingenommen wäle.

Diese überaus grosse Anzahl verschlungener und sich verHeehtender Nervenfïdehen, welehe man bei vollstänliger Färbung des Nervenapparats dureh Methylenblau wahrnehmen kann, macht es rein unmöglich, alle Verschlingmigen und Windungen, welche sic im Innenkolben des Körperchens bilden, zn beschreiben, ja selbst in Zeichmmg wiederzugeben. Cngeachtet dessen, dass ich hestrebt gewesen bin, in meinen Zeichnungen sowohl die Anzahl, als anch den Charakter der Anordnung der den Endapparat bildenden Nervenfäilen möglichst genat wiederzugehen, so gewilhen sie dennoch keine vollkommene Vorstellung von der Anzahl und dem fast bei jedem Körperchen gauz eigenthïmlichen Verlauf besagter Fiden, wic man sie in der Wirklichkeit wahrnehmen kann.

Gewölnhlich findet man an der Stelle der Absonderung der Nervenästchen und Färlen, welche an der Bildung des Endapparats Theil hal,en, Erweiterungen von drei-, viereckiger oder unregelmässiger Form (Fig. 4.1, 5, 6, 7,8 und 9), die Aestchen und Fiiden selbst aher erweisen sich in ihrem ganzem Verlauf mit varicösen Anschwellungen von der mannigfaltigsten Form spindelartig, rund, eckig n. s. w. - , wie auch von der verschiedenartigsten Grösse besetyt (Fig. 1-9), und sie unterscheiden sich in dieser Beziehung nicht von den oben beschriebenen Endanschwellungen. Die Grösse der Varicositaten steht, so viel ich beobachten kounte, in einer gewissen Beziehung zur Frische der zur Untersuchung verwendeten Hant: in dem Falle, wenn letztere $1 /:-1$ Stunde wach dem Tode aur Färbung der Nerven cutnommen war; crsehienen die meisten Nerenästchen fast roll- 
Die Nervenendigungen in der Haut der đusseren Genitalorgane etc. 597

ständig glatt und nur sehr wenige ron ihnen hatten varicöse Anschwellungen.

Bei Anwendung starker Objective kann man leicht constatiren, dass jede varicöse Anschwellung aus einem Centralfaden besteht, der durch den Axentheil der Anschwellung hindurchgeht und aus der die Centralfäden umgebenden peripherischen Schicht (Fig. 24). Der Centralfaden vereinigt in der Art von mehr oder weniger langen Ueberbriickungen eine ganze Reihe varicöser Anschwellungen, wird durch Methylenblau sehr intensiv gefürbt und besteht, meiner Meinung nach, je nach der Dicke des betreffenden Nervenästchens, aus einer Fibrille oder einem Bündel von Nervenfibrillen, während die den Centralfaden an der Stelle der varicüsen Anschwellungen umgebende periphere Schicht unter dem Einfluss von Methylenblau eine sehr schwache Färbung annimmt und nichts anderes darstellt als eine Interfibrillarsubstanz. Letztere lagert sich in uberlebendem Nervengewebe als eine gleichmüssig. dünne Schicht an der Periphcrie aller Nervenästchen und Fäden $a b$, während sie mit dem Eintritt der postmortalen Veränderungen wahrscheinlich ein wenig aufquillt und in abgesonderten Klümpchen sich ansammelt, welche sich auf dem Verlaufe der genannten Aestchen vertheilen und ihnen ein charakteristisches Bild geben. Häufig erscheint ein oder das andere Aestchen auf eine zienlich weite Strecke hin verdickt und dabei mit feinsten kleinen Auszackungen besetzt, welche dem Anscheine nach nur aus Interfibrillarsubstanz bestehen.

Erweiternngen verschiedener Art und Form, die sich an der Stelle der Theilung. der Nervenästchen befinden, sowohl wie die varicösen Anschwellungen können bei unvollständiger Tinction des Nervenapparates irgend eines Körperchens meiner Meinung nach als die oben beschriebenen Endanschwellungen erscheinen.

Ausser dem Nervenendapparate befindet sich in dem Innenkolben jedes Körperchens noch eine geringe Menge einer besonderen, aller Wahrscheinlichkeit nach, halbflïssigen, feinkörnigen Substanz, welche alle freien Zwischenräume ausfüllt, die zwischen den Schlingen der Nervenfüdlen des Endapparats vorhanden sind. Unter dem Einfluss langdanernder Einwirkung des Methylenblaus fürbt sich die bezeichnete feinkörnige Substanz in den meisten Fällen mehr oder weniger intensiv blau, wobei die Färbung derselben, so viel ich beobachten konnte, zu der Zeit be- 
gimnt, wann dic Nerrentäden des Endapparats bereits die Fähigkeit verloren haben, die Färbsubstanz in sich aufzunchmen; gleichzeitig. mit der Färbung der körnigen Sulostanz des Immenkolbens färben sich auch viele Korne der Hülle, und darauf schliesslich die letztere selbst, wodurch diss ganze Kürperchen in solchen Fällen blau tingirt erscheint.

Anlangend dic Nervenendigungen in den znsammengesetzten Genitalnervenkörperchen, in welehen der Innenkolben aus einigen mit einander in Verbindung stehenden Abtheilungen besteht, so geht gewöhnlich das eine oder das andere Nervenästehen aus einer Abtheilung des Kolbens in eine andere, benachbarte Abtheilung über, in welcher sie in oben beselniebcner Weise endigt, oder der Axencylinder der Nervenfaser theilt sich vor seinem Eintritt in das Körperchen in einige Iestehen, von welehen jedes in eine besondere Abtheilung des Innenkolbens des zusammengesetzten Körperchens eindringt.

Falls das zusammengesetzte Körperchen mehrere Nervenfasern erhält, tritt gewöhulich ein Axencylinter einer der Fasern in jede Abtheilung des Innenkolbens, wo er sich verzweigt. Aber so viel ich beobachten komute, - und es ist einerlei, ob num jecle der Abtheilungen des Innenkolbens ihre selbststiindige Nervenfaser oder cin Aestchen empfüngt, las aus der Theilung des Axencylinders noch ror dessen Eintritt in das Kärperchen entstanden ist, - vereinigen sich überhaupt alle Nerveneutlapparate, die in den verschiedenen Abtheilungen abgelagert sind, stets mit einanter durch mehr oder weniger dicke Nervenfiden, welche aus ciner Abtheilung des Kolbens in die andere übergehen.

Es kommt lange nicht immer vor, class alle Nervenfäden, die sich im Innenkolben irgend eines Körperchens verzweigen, auch in demselben endigen: einige (2-3) von ihnen dringen durch die Hülle des betreffenden Körperchens ein und begeben sich zu einem anderen oder zu meltreren in der Nähe befindlichen Körperchen; nachdem sie in den Innenkolben dieser letzteren eingedrungen, zerfallen die bezeichneten Aestchen in abgesonderte Fäden, welche sich mit den Nervenästchen und Fäilen des Endapparats des betreffenden Körperchens vereinigen. Auf sulche Weise wird mit Hülfe der eben beschriebenen Aestchen eine gemeinschaftliche Verbindung zwischen den nächsten und den von einander entfernten Körperchen hergestellt. 
Die Nervenendigungen in der Haut der äusseren Genitalorgane ete. 599

Bei Berücksichtigung aller eben mitgetheilten Beobachtungen, welehe auf mögliehst genane Untersuchungen einer zahlreichen Menge von Prïparaten begrindet sind, meine ich, dass eine grosse Menge von Nervenästchen und Fäden, die aus der Theilung ler $\Lambda$ xencylinder der $\mathrm{Ner}$ venfasern im Innenkolben der Körperehen entstandensind, sich windend und mit ein ander verflechtend, schliessliel ein seltreomplieirtes und dabei geschlossenes system mit einander verbundener Nervenfäden bildet (Fig. A). Ein jedes soleles ahgesondertessystem, in welchem die Axencylinder einer oder mehrerer Nervenfasern endigen, steht mittelst der oben beschriebenen anastomosirenden Fäden in unmit telbarer Verbindung mit anderen eben solchen systemen - mit Endapparaten (s. umsteh. Fig. A).

b) Die Nervenkörperchen (Endkolben - W. Krause) (Fig. 12-18). Ausser den Genitalnervenkörperchen liegen in der Hant der imneren Lamelle des Praeputium, Frenulum praeputii, der Glans penis, so wie anch in der Haut der Fossa navieularis des Menschen noch besondere Nervenapparate, welche nach der Art rer in ihnen endigenden Nerven, nach ihrer Lage u. s. w. am meisten Achnlichkeit haben mit den von $\mathrm{mir}$ beschriebenen Körperchen im Gefïsse enthaltenden Rande der Hornhant und in der Bindehant des Anges ${ }^{1}$ ).

Sie liegen sehr oberflachlich - entweder im Gewebe der Wärzchen der Hant oder an deren Basis (Fig. 1), fast unmittelbar unter dem Epithel, und zudem in ziemlich beträchtlicher Menge, so dass auf ein $1 \mathrm{qmm}$ annähernd 4-20 Körperchen zu liegen kommen; nur in seltenen Fällen findet man dieselben neben den Genitalnervenkörperchen.

Die Grösse der zu beschreibenden Endapparate ist im allgemeinen eine viel geringere, als die der Genitalnervenkörperchen, während ihre Gestalt eine runde, birnförmige, eiförmige oder ovale zu sein pflegt.

1) Die Nervenendkörperchen (Endkolben, W. Krause) in der Cornea und Conjunctiva bulbi des Menschen. Archiv f. mikrosk. Anatomie, Bd. XXXVII, p. 602-619. 
Zu dem Bestande dieser Körperchen gehört cine feine bindegewebige Hïlle, welche durch zwei, drei Hiintehen - Kapseln gebildet wird, zwischen denen grosse, runde oder ovale Kerne

Fig. $\mathrm{L}$.

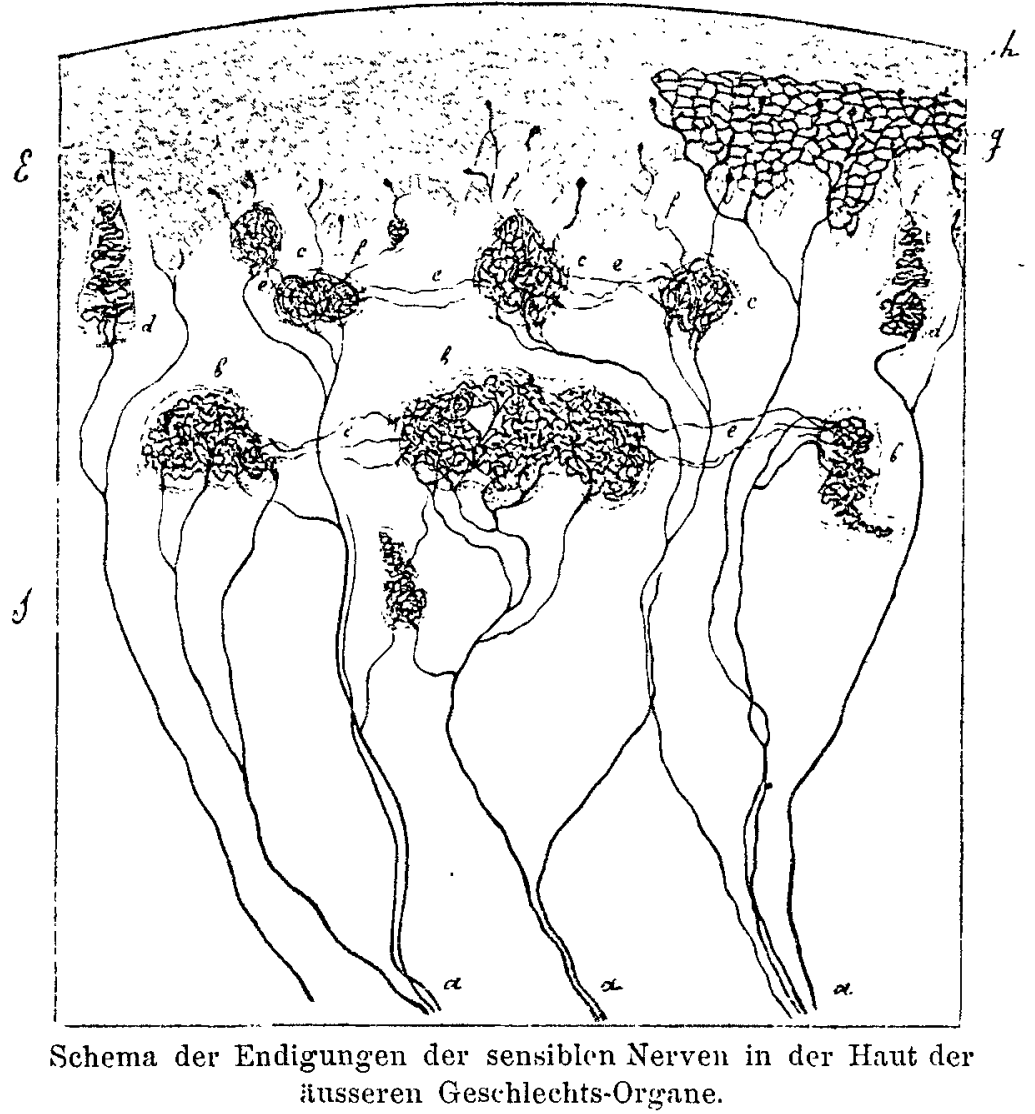

a) Nervenfasern; b) Genitalnervenkörperchen; c) Nerrenendkörperchen (Endkolben, W. Krause); d) Meissner'sche Körperchen; e) Nervenfäden, welche die Nervenendapparate verbinden; f) intracpitheliale Nervenfäden; g) intraepitheliales Nervennetz; h) Nerventiiden, welche mit knopfförmigen Verdickungen endigen. E) Epithelium; S) Cutis.

liegren, die zu den abgeplatteten Zellen gehören. Die innerste Kapsel der Hülle des Körperchens begrenzt die Höhlung (den Imenkolben), wobei die zur Seite der letzteren mewendete Obelfläche derselben, :̈lhnlich wie es bei den Genitaherrenkïrperchen 
Die Nervenendigungen in der Haut der äusseren Genitalorgane ete. 601

der Fall ist, mit einer Schicht abgeplatteter Zellen besetzt ist, deren Kerne mehr oder weniger weit in die Höhlung des Körperchens hineiuragen.

In den allermeisten Fällen empfängt jedes Körperchen ein, zwei, und nur sehr sclten drei markhaltige Nervenfasern (Fig. 12-18), oder aber eine markhaltige Nervenfaser theilt sich zuror in einige ( $5-6$ und mehr) lange orler kure Aestehen, von denen die einen eine Markscheide besitzen, wïhrend andere in ihrem ganzen Verlauf derselben entbehren, yobei jedes Aestchen in ein abgesondertes Körperchen eindringt (Fig. 12-18). Oft theilt sich irgend ein beliebiges Aestchen abermals in zwei Aestchen, welche in zwei verschielenen Körperchen endigen (Fig. 12). Die eben bezeichneten Nervenfasern und $\Lambda$ estchen, welche die Richtumg nach den Körperchen nehmen, schlingen sich, so viel ich beobachten konnte, selten vor ihrem Eintritt in den Innenkolben um die Oberfläche des Körperchens, sondern begeben sich gewöhnlich direct zu beiden oder zu einem seiner Pole, wobei die Schwann'sche Scheide der Nervenfasern mit der Hülle des Kürperchens zusammenfliesst, während die Marksubstanz derselben in einiger Entfernung von der Hülle des Körperchens verschwindet.

Der Axencylinder der Nervenfaser, nachdem er an die Hülle des Körperchens herangetreten, durchdringt dieselbe und theilt sich sogleich nach seinem Eintritt in den Innenkolben in einige (2-3 und mehr) varicöse Aestchen; nur in verhältnissnässig seltenen Fällen findet diese Theilung des Axencylinders früher, in km"zer Entfernung vom Körperchen statt. Die besagten Aestchen beginnen sich bald darauf in der Höhlung des Körperchens auf solche Weise zu winden, dass jedes derselben nach verschiedenen Richtungen hin eine Menge schlingenartiger Windungen macht, die sich mit einander und mit den von anderen Aestchen gebildeten Windungen durchkreuzen, wobei sie unterwegs ihrerseits wiederum in einige feinere Aestchen zerfallen (Fig. 12-18). Die letzteren winden sich aufs nene, schlängeln und durchflechten sich mit einander in der allermannigfaltigsten Weise, theilen sich oft aufs neue in eine gewisse Anzahl in ähnlicher Art sich windender Fäden und endigen schliesslich als stumpf geschlossene Schlingen (Fig. 12-18).

In Folge dieses eigenartigen Verlaufs der Nervenüstchen Arehiv f. mikrosk. Anatomie. Bi. 41 
und Fäden in Innenkolben erbält der ganze Endapparat, wie aus Fig. 12-18 ersichtlich, das charakteristische Ansehen eines Knäiuels, der aus auf verschiedene Weise inber einander gewickelten Fäden besteht, welche die ganze Höhlung des Kolbens ausfüllen. Bei allmählicher Aenderung der Focaldistan\% beobachtet man an den Flächenprïparaten anfangs Schlingen, die an der Peripherie des Imnenkolbens belegen sind, darauf eine Reihe Schlingen, welche tiefer liegen u. s. w., wobei in Folge dessen, dass die varieösen Aestchen und Fäden, welche Schlingen bilden, sich gewunden haben, man sie bei einer gewissen Focaldistanz im optischen Durchschnitt, als Endanschwellungen von verschiedener Grösse und Form erblickt; aber man braucht nur die Focaldistanz zu ändeın, um sich davon zu überzeugen, dass die besagten Anschwellungen in der That nur optisehe Durehsehnitte von Nervenfäden oder von varicösen Anscliwellungen sind. Freilich, in dem Falle, wenn nicht alle Nervenfäden, welche den Endapparat bilden, mit Methy̆lenblau gefärlbt sind, scheinen einige ron ihnen wirklich frei $z \mathfrak{u}$ endigen, wie dieses anch bei den Genitalnervenkörperchen erwiilnt wurde.

Je grösser das Endkörperchen ist, desto melır zusammensesetzt pflegt gewölnlich ler rou den Nervenästehen und Fäden im Innenkolben grebildete Knäitel zu sein, d. h. die Nervenfäden sind während ihres sich sehlängehden Verlauts häufigen Theihurgen unterworfen und billen eine grössere Anzahl von Schlingen (Fig. 13 und 14). In kleinen Körperchen theilt sich in Gegentheil der Axencylinder, nachdem er zuvor einige spiralförmige Windungen gemacht, oder sogar gleich nach seinen Eintritt in das Körperchen, in 2-3 Aestclien, welche sich mebr oder weniger winden und mit blinden Schlingen endigen (Fig. 12, 16, 17 und 18). Oft werden von den Nervenästchen feinc und dicke, mit grossen variciösen Anschwellungen besetzte Seitenfiiden abgesondert, welche zur Verbindung der nahe liegenden Aestchen dienen.

Ausser allem Gesagten erregt an Flächenpräparaten und sogar an Schnitten mit möglichst vollständiger Färbung der Nerven eine interessante Thatsache die Aufmerksamkeit, nämlich, dass sich von den Endapparaten vieler Körperchen Nervenfäden verschiedener Dicke abtheilen, welche die Hülle durchdringen und ans dem betreffenden Körperchen austreten (Fig. 17 und 18B). 
Die Nervenendigrngen in der Haut der ausseren Genitalorgane etc. 603

Das fernere Schicksal solcher Fäden, die sich auf den Endapparat eines und desselben Körperchens beriehen, pflegt ein verschiedenes zu sein: nachdem die einen von ihnen eine grössere oler kleinere Strecke weit verlaufen sind, treten sie in $d$ en Innenkolben eines beliebigen benachbarten Körperchens ein und dienen entweder ganz zur Bildung eines besonderen Endapparats, oder sie a lustomosiren nur mit den Nervenfäden des letzteren (Fig. 17d und e), während andere Fäden hä ufig sieh anfangs in einer gewissen Ausdehnung unter dem Epithel ausbreiten, darauf sich zuweilen dichotomisch theilen und dann in as Epithel eintreten, wo sie sich zwischen den Epithelzellen winden, um schliesslich indentieferen Schichten desEpithels mit grossen ovalen, conischen oder birnförmigen Anschwellungen freizu e n d i ge n (Fig. $17 \mathrm{~b}$ und e; Fig. 18B). Vermittelst der letzteren steht der Nervenendapparat des Körperchens in einer gewissen Beziehung zu dem Epithel.

Nach meinen neuesten Beobachtungen werden Nervenästchen ähnlicher Art, die frei zwischen den Epithelzellen endigen, auch yon den in der Conjunctiva bulbi belegenen Nerrenknäueln der Enrlkolben von W. Krause abgegeben und es ist sehr wahrscheinlich, dass dergleichen Aestchen auch in den Genitalnervenkörperchen vorhanden sind, nur hält es wegen der tieferen Lagerung der letateren in der Haut weit schwerer, sie zu verfolgen.

Auf solche Weise besteht der Hauptunterschied zwischen den eben beschriebenen Nervenendapparaten und den Genitalnervenkörperchen darin, $d$ ass erstere mehr oberfläch. lich, fast unmittelbar anter dem Epithel liegen, ferner darin, dass die Nervenästchen und Fäden, welche sich im Innenkolben verbreiten, in dem. selben eine Menge schlingenartiger Windungen machenundnichtineinesouberausgrosseAnzahl sich vielfältig theilender Fäden zerfallen, wie das bei den Genitalnervenkörperchen der Fall ist (Fig. A). Indessen kann man im allgemeinen sagen, dass ausser den oben aufgezillilten keine anderen deutlich hervortreteuden Unterscheilungs-Merkmale zwischen den Endkolben und 
den Genitahervenkürperehen cxistiren; in Folge dessen kann man sie unter gewissen Bedingungen kaum, oder jedenfalls sehr schwer von einander unterscheiden.

c) Die Meissner'schen Körperchen (Fig. 19) trifft man in der Hatut der Innenlamelle des Praeputium, der Glans penis und der Clitoris in sehr beschränkter Anzahl an; sie liegen gewöhnlich sowohl in den grossen als auch in den kleinen Papillen und nehmen diese of vollständig ein, so dass sich die Oberflüche des Körperchens fast unnittelbar an das die Papille umgebende Epithel anlegrt. So viel ich beohachten komnte, trifft man die Me issner'schen Körperchen häufiger in der Hant des Praeputium, Collum und der Corona glandis, als in der Glans penis; in der Hant der Fossa navieularis findet man sic überhaupt nicht.

An Fläelenpräparaten des Pracpntinm und der Glans penis, gleich wie anch an den Schnitten der Traut der Finger und Fuisse des Menschen, hat man die Mögliehkeit, die Nervenendigung in den Mcissner'schen Körperehen genau zu erforsehen, wobei ich dic Möglichkeit hatte, meine früheren Beobachtungren ') nochmals zu controliren und mich von ihrer Richtigkeit zu iibcrzeugen.

Bei Untersuchung der bezeichneten Präparate kann man, besonders bei vorausgegangener Färbung durch Methylenblau, eine ziemlich dicke Bindlegewebshülle der Meissner'schen Körperchen mit einer bedeutenden Menge in derselben alygelagerter Kerne von runder oder ovaler Form beobachten; ein grösserer Theil dieser Kerne gehört zu den platten Zellen der Bindegrewcbshülle des Körperchens, während der übrige Theil zu den Kernen der Schwann'schen Scheide der Nervenfasern in Beziehung. stelit, die sich oft an der Oberfäche des einen oder des anderen Körperchens winden. Gewöhnlich lagern sich einige Kerne quer und schräg, andere parallel zur Längsaxe jedes Körperchens.

Die imnere Oberffäche der Hülle, welche zur Höblung zum Innenkolben - des Meissner'sehen Körperchens gerichtet ist, ist nach meinen Beobachtungen mit ebensolchen platten Zellen

1) Die Nervenendigungen in Meissner'schen Tastkörperchen. Internat. Monatsichrift f. Anat. n. Phys. 1892, Bd. IX, H. 2. 
Die Nervenentigungen in der Haut dur äusseren Genitalorgane ete. 605

besetzt, wie sie sich in der Hülle des Körperchens befinden, wobei die Kerne dieser Zellen sich dann und wann zugleich mit den Nervenästchen durch Methylenblau färben und in solchen Fällen sehr leicht für Terminalanschwellungen gehalten werden können. Jedes Körperchen empfängt je nach seiner Grösse ein, zwei und zuweilen auch drei Nervenfasern, welche grösstentheils in das Körperchen an dessen unterem Pol eindringen odcr, in anderen Fällen macht die Nervenfaser um das Kärperehen einige Windungen, durchdringt erst dann die Bindegewebshiille an irgend einer Stelle der Oberfläehe des Körperchens in grösserer oder geringerer Entfernung von dessen unterem Pole und tritt darauf in den Innenkolben ein.

Wenn das Körperchen aus einigen Läppchen besteht, so empfüingt in solchen Fällen jedes Läppchen eine abgesonderte Nervenfaser oder ein, zwei Nervenfasern zerfallen zuvor an rlei Basis des Körperchens in eine gewisse Anzahl Aestchen, welche sich zu den Läppchen begreben.

Die Nervenfasern verlieren ihre Markscheide neben oder an der Oberfläche des Körperchens selbst oder häufig in beträchtlicher Entfernung von demselben. Nicht selten theilt sich irgend eine markhaltige Faser nahe bei lem Meissner'schen Kibrperchen an der Stelle der Ranvier'schen Einschnürung in einige Aestchen, von denen die einen ihre Markscheide verlieren, wïhrend andere dagegen dieselbe bis dicht zur Hülle des Körperchens selbst bewahren.

Nachdem der Axencylinder der Nervenfaser in das Innere des Meissner'schen Körperchens cingetreten, wie dicses von mir bereits des genaneren beschricben worden ist, rerläuft er, indem er sich an den Axentheil oder an die Pcripherie des Innenkolbens hält, mit einer kleinen Windung zu dem entgegengesetzten Pol des Körperchens, wobei er unterweg's Scitenästehen von verschiedener Länge und Dicke aussendet und schliesslich sclbst in einige Aestehen zerfïllt (Fig. 19). Alle Aestchen, welche durch allmähliche Theilung des Axencylinders entstanden sind, zerfallen ihrerseits bald wiederum in feinere Aestchen und Fiiden, welche sich wellenartig krïmmen und im Innenkolben eine gewisse Inzahl fast kreisförmiger Windungen bilden, die sich bald an der Peripherie, bald in den tieferen Theilen der Höhlung des Körperchens lagern und sich zugleich mit anderen, anf ähuliche Weise 
gewundenen Aestchen kreuzen (Fig. 19). Die soeben beschriebenen Aestchen vereinigen sich mit einander und endigen schliesslich mit Schlingen, wobei die von ihnen gebildeten Windungen quer, und ein wenig schräg zur Längsaxe des Körperchens lieg’en und sich nur zuweilen an der Spitze desselben mehr oder weniger parallel zur bezeichneten Axe lagern (Fig. 19); in den zusammengesetzten Körperchen künnen die Winclungen jedes Läppchens quer, schräg oder parallel zur gemeinsehaftlichen Längsaxe des betreffenden Körperchens gerichtet sein. Gewöhnlich pflegen die Nervenästchen und Fäilen, welche ren Endapparat billen, mehr oder weniger abgeplattet und mit varicösen Anschwellungen von verschiedener Grösse und Form besetzt zu sein, welehe bei einer gewissen Focaldistanz oder in Folge nicht vollständiger Tinction eines oder des anderen Aestehens, wie es mir scheint, Anlass geben können, die Existen\% freier Endigungen in der Art von Terminal-Inschwellungen anzmehmen.

Die kleinen Zwischenrïume, welche im Innenkolben der Körperchen von den Nervenfäden des Eudapparats nicht eingenommen werden, sind mit einer leicht kïrnigen Substanz angefüllt, die sich gleich wie die Kerne der Hülle nur nach langandanernder Einwirkung des Farbstoffes (des Methylenblau) färben, wobei das ganze Körperchen in solchen Fällen eine mehr oder weniger intensiv blane Fürbung amimmt. Die Anwesenheit irgend welcher Zellen im Innenkolben, al)gesehen von der oben beschriebenen körnigen Substanz, konnte ich nicht constatiren.

Wenn wir die Beschreibung der Nervenendigungen in den verschiedenen Nervenapparaten - den Genitalnervenkörperchen, den Endkolben und den Meissner'sehen Körperchen -, welche in der Haut der äusseren Genitalorgane belegen sind, vergleichen und zugleich die beigegebenen Zeichnungen betrachten, so müssen wir zu dem Schluss kommen, dass zwischen ihnen kein wesentlicher Unterschied besteht: in allen a u f e fïhrten Apparatentretendie Axencylinder der markhaltigen Nervenfasernin die Höhlung-den Innenkolbendes betreffenden $K \ddot{j} r$ perchens ein, zerfallen in derselben in eine gewisse Anzahl varicöser Aestchen und Faden, welche wïrend ihres Verlants 
sich winden, eine Menge spiralartiger Krïmmungen machen, sich vielfältig theilen, vereinigen, durchkreuzen und in verschiedenartiger Weise mit einander verflechten, und schliesslich ein ganzes System von mit einander verbundenen Sehlingen oderein sehrverwickeltesund dichtes Nervennetz bilden. Der ganze Unterschied, der zwischen den Körperchen der verseliedenen Typen existirt, beschränkt sich nur anf die verschiedene Form, Grös e und Lage derselben in der Hat und beruht hautsachlich a uf der Anzahl und Vertheilung der Nervenastehcn, welche durch die allmähliche Theilung der Axeneylinder in der Höhlung-dem Innenkolbendes $\mathrm{Korperchensentstandensind.} \mathrm{Indieser} \mathrm{letz-}$ teren Bezichung nehmen, meiner Ansicht nach, die Genitalnervenkörperchen die erstestelle ein nnd wir müsen sie zu den am meisten zusammengesetzten Endapparaten reehnen, daraf folgen die Meissner'sehen Körperchen und endlich mus manden Endkolben, namentlichaber denkleinen, bei welehen der Nervenendapparat a s einem oder aus mehrerenspiralartig gew ulenen Fäden besteht, die letzte stelle einr äumen.

Schliesslich haben fast alle Terminal-Körperchendas mit einander gemein, dass vondem Nervenapparateder Körperchen eines jeden Typus sich eine gewisse $A$ nzahl Nervenfäden absondert, von welchen die einen in das Epithel eindringen undmit knopfförmigen Anschwellungen endigen, während die anderen Fäden zur gemeinschaftlichen Verbindung der Endapparate des betreffenden Typus von Körperehen dienen.

Das dem Text beigefügte Schema (Fig. A) griebt ein Bild von den Endigungen der sensiblen Nerven in der Hant der äusseren Geschlechtsorgane.

Die Nervenendigungen im Epithel (Fig. 20). Die Nervenstämmchen von verschicdener Dicke, welehe, wie wir vorhin gesehen bahen, in der tief'en, lockeren Schicht der Haut 
liegen, bestehen aus markhaltigen und aus marklosen Fasern, wobei eine bedeutende Anzahl der ersteren in den oben beschricbenen Endapparaten endigt, während die iibrigen markhaltigen Fasern, nachdem sie sich von den Nerrenstämmchen abgesondert haben, schräg. odler senkrecht zum Epithel hin verlaufen und, nach Verlust ihrer Markscheide, in abgesonderte Aestchen zerfallen. Die letzteren breiten sich anfangs auf einer geringen Strecke nuter dem Epithel aus, biegen daranf nach oben lin um und dringen fast in verticaler Richtmug in das Epithel ein (Fig. 20). Alle soeben bezeichneten intraepithelialen testehen krümmen sich, wie dieses an Schnitten und Flächenpräparaten ersichtlich ist, mehr oder weniger zwischen den Epithelzcilen, wobei sie unterwegs naeh verschiedenen Richtungen feine Scitenästchen aussenden, um schliesslich, nachdem sie die oberflächlichen Schichten des Epithels erreicht haben, strahlenförmig in eine grössere oder kleinere Anzahlıl sehr feiner Aestchen und varicöser Fäden zu zerfallen. Die cinen der oben bezeichneten Aestehen und Fäden winden sich anf verschicdene Weise zwischen den Epithelzellen, zerfallen beständig in eine Nenge feiner varicöser Fädlen und bilden, nachdem sie sich mit anderen ähnlichen Fäden vereinigt haben, ein feinmaschiges Nervennetz. (Fig. 20); andere Aestchen verlaufen gleich den ersteren zuerst sich windend zwiselien den Epithel\%ellen und endigen darauf in verschiedener Entfernung von der freien Oberfläche der Epitheldecke mit knopfförmigen Anschwellungen (Fig. 20 b).

Die marklosen Nervenfasern (Fig. 21, 22, 23) liegen zusammen mit den markhaltigen Fasern entweder, wie oben gesagt, in den Nervenstämmchen, oder sie bilden abgesonderte mehr oder weniger dicke Stämmelen. Die ersteren sondern sich bald von den markhaltigen Fasern ab und bilden, indem sie sich mit den ausschliesslich aus marklosen Fasern bestehenden Stämmchen verflochten haben, in der tiefen, lockeren Schicht der Haut ein feinmaschiges Geflecht. Von diesem Geflecht gehen Aestchen zweifacher Art aus: die einen verlaufen zu den Blutgefässen, die anderen treten in die obere, mehr compacte Schicht der Haut ein und bilden in derselben, indem sie allmählich in feine Aestchen und Fäden zerfallen, ein dichtes Geflecht (Fig. 21). Die Schlingen dieses Geflechts haben eine nnregelmässige, vieleckige Form, wobei einige von ihnen unmittelbar 
Die Nervenendigungen in der Haut der äusseren Genitalorgane etc. 609

unter dem Epithel liegen und an Fläehenpräparaten in derselben Focaldistanz sehr deutlich hervortreten (Fig. 21), bei welcher auch die Epithelzellen der allertiefsten Schicht - der Keimschicht - genau wahrnehmbar sind. Gewöhnlich entstehen an denselben Stellen des Geflechts, wo einige, Schlingen bildende Aestchen zusammentreffen, knotige Verdickungen, in welchen grosse ovale Kerne liegen (Fig. 21 und 22); in Folge dessen erhält das ganze Geflecht ein besonderes, charakteristisches Anschen. Aehnliche Kerne trifft man auch in Verlanfe der einzelnen Nervenästchen. Von den Schlingen des unterepithelialen Geflechts, welches durch die marklosen Fasern gebildet wird, sondern sich, soviel ich beobachten konnte, feine, in die Tiefe des Epithels gehende Aestchen ab and zerfallen in ihm in eine Menge feiner Nervenfülen; die letzteren betheiligen sich zusammen mit den Fäden, die aus der Theilung der markhaltigen Fasern entstanden sind, aller Wahrscheinlichkeit nach, an der Bildung des intraepithelialen Nervennetzes. Was diejenigen Nervenaistehen anbetrifft, welche, wie oben gesalgt, zu den Blutgefässen hin verlaufen, so umspinnen sie die bindegewebige Hant der Arterien (Fig. 23) und Venen als ein dichtes Nervengeflecht'). Von diesem Geflecht geht wiederum eine gewisse Anzahl sehr feiner Aestchen und varicöser Fädlen aus, welche in der Muskelhaut der Gefässe in Form eines Nervenendnetzes endigen.

Alles von mir in Bezug auf die Nervenendigungen in der Hant der äusseren Genitalorgane des Menschen Gesagte bezieht sich in gleicher Weise anch auf die Hant des Praeputium und der Glans penis weisser Mäuse, Ratten und Albino-Kaninchen, wie dieses meine späteren Beobachtungen dargethan haben.

1) An der Bildnng dieses Geflechts nehmen auch einige markhaltige Nervenfasern Antheil. 


\section{Literatur.}

1. L. Fick, Lehrbuch der Anatomie des Menschen. Leipzig. 184ă.

2. Kölliker, Mikrosk. Anatomic. II. 2. $18 \overline{\mathbf{s}}$.

3. W. Krause, Ueber die Nervenendigungen. Zeitschr. t. rationelle Vedicin. Bd. V. 1858.

- - Dic terminalen Körperchen der eintach sensiblen Nerven. 1860.

4. Kölliker, Gewebelehre. 4. Antl. 1863.

๖. Dr. Polle, Die Nerven-Verbreitung in den weibl. Genitalien. 1865.

b. Tomsa, Ueber den peripherischen Verlauf und Endigung des Axencylinders in der Haut der Glans penis. Sitzungsber. der $k . k$. Akad. d. W. Bd. 51. 1865.

7. W. Krause, Ueber die Nervenendigung in der Clitoris. Göttinger Nachrichten. 21. April 1866.

- -, Ueber die Nervenendigung in den Geschlechtsorganen. Zeitschrift ft. rat. Medicin. Bd. XXVIII. 1866.

8. W. Finger, Ueber die Endigungen der Wollustnerven. Zeitsehr. f. rat. Nedicin. Bd. XXVIII. 1866 .

9. Bense, Zeitschr. f. rat. Mediein. 3. Reihc. Bd. XXXIIr. 1868.

10. Axel Key und G. Retzius, Studien in der Inatomie des Nervensystems und des Bindegewehes. Bd. II. Stockholm. 1876.

11. Iz $r_{1}$ uierdo, Beitrige zur Kenntniss der Endigung der sensiblen Nerven. Strassburg. 1879.

12. Fr. Yerkel, Ueber die Endigung der sensiblen Nerven in der Haut der Wirbelthiere. Rostock. 1880.

13. W. Krause, Archiv f. mikrosk. Anatomie. Bd. XIX. 1881.

14. H. Aronson, Beiträge zur Kenntniss der centralen und peripheren Nervenendigungen. Inaug.-Diss. Berlin. 1886.

15. G. Schwalbe, Lehrbuch der Inatomie der Sinnesorgane. Erlangen. 1887.

16. Kölliker, Handbuch der Gewebelehre. 6. Aufl. 1889.

17. G. Retzius, Ueber die Endigungsweise des Nerven in den Genitalnervenkörperchen des Kaninchens. Internat. Monatsschrift für Anatomie und Physiologie. Bd. VII. 1890.

18. P. Schiefferdecker und A. Kossel. Gewebelehre. Bd. II. 1891.

\section{Erklärung der Abbildungen anf Tafel XXXII u. XXXIII.}

Alle Zeichnungen sind mit einer Camera-lucida von Oberhäuser vou Priparaten aufgenommen, welche durch Methylenblau getiirbt und 
Die Nervenendigungen in der Haut der äusseren Genitalorgane ete. 611

mittelst einer Ammoniumpikratlösung oder einer Mischung derselben mit Osmiumsäure fixirt waren.

Fig. 1. Quersehnitt der Haut der Glans penis des Menschen. a) Epithel; b) Cutis; c) Nervenstämmchen; d) Genitalnervenkörperchen; e) Endkolben. Reichert's Obj. 4.

Fig. 2 u. 3. Genitalnervenkörperchen aus der Glans penis des Menschen. a) Hülle eines Nervenstïmmchens; b) Hülle eines Körperchens mit in derselben abgelagerten Kernen platter Zellen; c) Axencylinder von Nerventasern, welche sich im Innenkolben des Körperchens verzweigen. Reichert's Obj. 8 a ul. 6 (Fig. 3).

Fig. $4 \alpha$ u. B. Genitalnervenkörperchen aus der Glans penis des Menschen mit an der Oberfläche derselben sich schlängelnden markhaltigen Nervenfasern. Die Nervenfasern, deren Axencylinder rosa-violett (an der Stelle der Ranvier'schen Einschnürungen dunkel-violett) dargestellt sind, haben sich auf der dem Beobachter zugewendeten Seite des Körperchens ausgebreitet. a) Hülle; b) markhaltige Nervenfasern; c) markhaltige Faser, deren Axencrlinder sich im Innenkolben des Genitalnervenkörperchens verzweigt; (d) zwei feine Aestchen, welche sich rom Axencylinder an der Stelle der Ranvier'schen Einschnürung absondern und in einem kleinen Körperchen endigen. Reicherts Obj. 6.

Fig. 5-8. Genitalnervenkörperchen verschierlener For'm aus der Glans penis des Menschen. a) Markhaltige Nervenfasern; b) Hülle. Fig. 5 n. 6 sind bei einem Obj. 8 a geezeichnet, die uibrigen bei Reichert's $0 \mathrm{bj} .6$.

Fig. 10-11. Genitalnervenkörperchen aus dem Praeputium einer weissen Ratte. a) Markhaltige Norvenfasern; b) anastomosirende Nervenfäden; c) Hülle. Fig. 10 ist bei Obj. 4 und Fig. 11 bei Obj. 6 Reichert's gezeichnet.

Fig. 12. Nervenendkörperchen (Endkolben) aus der Glans penis des Mensehen. a) Markloses Nervenästchen, das aus der Theilung: des Axencylinders einer markhaltigen Faser entstanden ist. Reichert's Obj. 8a.

Fig. 13-16. Nervenendkïrperchen (Endkolben) aus dem Praeputium (Fig. 13) und der Glans penis des Menschen. a) Markhaltige Nervenfasern. Fig. 13-14 bei Obj. 6 abgezeichnet, die übrigen bei Reichert's Obj. $8 \mathrm{a}$.

Fig. 17. Endkolben aus der Fossa navicularis des Menschen. a) Markhaltige Nervenfaser, deren Axencylinder in der Höhlung des Körperchens einen Nervenknäuel bildet. Aus dem Nervenknäuel gehen 4 Aestchen aus (b, $c, d$ und e), wobei zwei (b und c) zwischen den Epithelzellen mit knopfförmigen Verdickungen endigen, die zwei anderen ( $d$ und e) dagegen ganz zur Billung eines neuen Nervenknänels verwondet werden. f) Epithel, Reichert's Obj. \&a. 
Fig. 18A u. B. Endkolben aus der Glans penis einer weissen Maus. A) a) Markloses Aestchen, das einen cinfachen Nervenkniuel bildet. B) b) Markhaltige Nervenfasern, deren Axencylinder mit einem Nervenknäuel enligen. Aus dem Knäuel geht ein feines Nervenfadchen aus, das zwischen den Epithelzellen endigt. Querschnitt. Reichert's Obj. 8 a.

Fig. 19. Fin Meissner'sches Körperchen aus dem Praeputium des Menschen. a) Markhaltige Nervenfaser. Reichert's Obj. 8a.

Fig. 20. a) Nervenästchen, welehes durch Theilung des Axeneylinders einer markhaltigen Faser entotanden ist und zwischen deu Epithelzellen des Praeputium eines Kaninchens endigt; b) ein Endknöpfehen. Flaichenpräparat. Reichert's Obj. 6.

Fir. 21. Ein aus marklosen Fasern bestchendes Nervengeflecht der Haut der Glans penis des Menschen. a) Nervenstanmchen, von welchen die das Geflecht bildenden Aestchen (b) ausgehen; c) Epithel. Flichenpraiparat. Reichert's Obj. 4, halbherausgezogener Tubus.

Fig. 22. Nervenastchen, an deren Vereinigungsstelle sich eine knotige Verdickung mit einem Kern bildot. Glans penis des Meuschen. Reichert's Obj. 8n.

Fig. 23. Nervengeftecht um eine kleine Arterie, deren Wand ungefirbt blieb. Praeputium eines Kanimehens. Reichert's Obj. 6.

Fig. 24. Einige Aestchen cines Nervenendapparates. a) Centralfialen und die denselben ungebende Srhicht (b) der Interfibrillarsubstanz. Das Priparat bei Reichert's Obj. 6 abgezeichnet und die Zeichnung daraut um das Doppelte vergrössert.

\section{Neuroglia der Retina des Menschen.}

Von

\section{A. S. Dogiel,}

Professor der Histologie an der Universitit zu Tomsk (Sibirien). Dritte Mittheil ung ${ }^{1}$ ).

Hierzu Tafel XXXIV.

Zur Untersuchung der Neuroglia der Retina des Menschen hediente ich mich ansschliesslich der Fürbungsmethode von M.

1) Cf, Archiv f, mikrosk. Anatomie Bd. XXXVII und XL. 